\title{
Defect Characterization in Next Generation Laser Crystals
}

\author{
J. B. LeBret,* D. E. Eakins, ${ }^{*}$ M. G. Norton, ${ }^{*}$ D. F. Bahr, ${ }^{*}$ Xiaoming Li** \\ * Mechanical and Materials Engineering, Washington State University, Pullman WA 99164 \\ ** II-VI, Inc., 375 Saxonburg Blvd., Saxonburg PA 16056
}

Yttrium orthovanadate $\left(\mathrm{YVO}_{4}\right)$ single crystals are becoming increasingly popular for use in solid state laser devices. Nd-doped $\mathrm{YVO}_{4}$ has a larger stimulated emission cross-section and absorption coefficient than Nd:YAG [1,2]. One hurdle for growers of $\mathrm{YVO}_{4}$ is to be able to produce crystals of large diameter and length, a task that is made difficult by the tendency for the growth direction to fluctuate. Imperfections in the form of discolorations have been observed in $\mathrm{YVO}_{4}$ crystals, shown in Figure 1a. These and other as-yet uncharacterized defects result in the scattering of light, a detrimental effect that limits the laser efficiency. An unstable growth direction, inhomogeneous coloration, and light scatter may result from flaws in the crystals. Knowledge of the defects present in $\mathrm{YVO}_{4}$ may lead to optimization of growth parameters and achievement of large, high quality laser crystals.

Samples for TEM analysis were prepared by conventional mechanical methods followed by chemical thinning to electron transparency using a $1: 1$ solution of $\mathrm{H}_{2} \mathrm{SO}_{4}$ and $\mathrm{H}_{3} \mathrm{PO}_{4}$ at $180^{\circ} \mathrm{C}$. Specimen preparation was complicated by the propensity for cleavage along (100) and (010) planes. Figure $1 \mathrm{~b}$ is a scanning electron microscope image showing the cleavage surface of a $\mathrm{YVO}_{4}$ crystal. Each of the large terraces are (100) planes, the sharp parallel ledges are (010) planes. The atomic arrangement is different for the (001) planes, resulting in a much lower atomic density. Consequently, cleavage does not occur on these planes, which is evidenced by jagged ledges.

Dislocation structures, shown in Figure 1c, were observed in TEM samples prepared from a crystal containing extensive regions of discoloration. Due to the nature in which a dislocation strains the lattice, it is possible to orient the sample such that the path of the electron beam is not affected. At such an orientation the dislocation would appear to be "invisible" and the criterion $\mathbf{g} \bullet \mathbf{b}=0$ would necessarily be true, where $\mathbf{g}$ is the diffraction vector, and $\mathbf{b}$ is the burger's vector of the dislocation. Figure 1d was recorded at an orientation where the invisibility criterion was satisfied. The diffraction vector was determined to be [112]. Possible b vectors may then be chosen to satisfy the above criterion, namely [0리, [02 $\overline{1}],[20 \overline{1}],[\overline{2} 01],[1 \overline{1} 0]$, and [ $\overline{1} 10]$. Inspection of the unit cell model for $\mathrm{YVO}_{4}$ shows that directions of the type $<021>$ link nearest neighbor like atoms. Hence, the likely

burger's vector is $\frac{\mathrm{a}}{4}[0 \overline{2} 1]$. Studies are continuing to examine possible mechanisms for dislocation motion and at what temperatures the dislocations can be removed. The results of these studies will be presented [3].

References

1. A. W. Tucker et al., J. App. Phys. 47 (1976) 232

2. B. Q. Hu et al., J. Crystal Growth 226 (2001) 511 
3. Financial support provided by the Department of Defense ASD/C3I (N66001-00-C-6008) through a subcontract from VLOC Inc. Part of this work was performed at the EMSL, a national user facility located at Pacific Northwest National Laboratory, operated for DOE by Battelle.

$$
\text { a }
$$
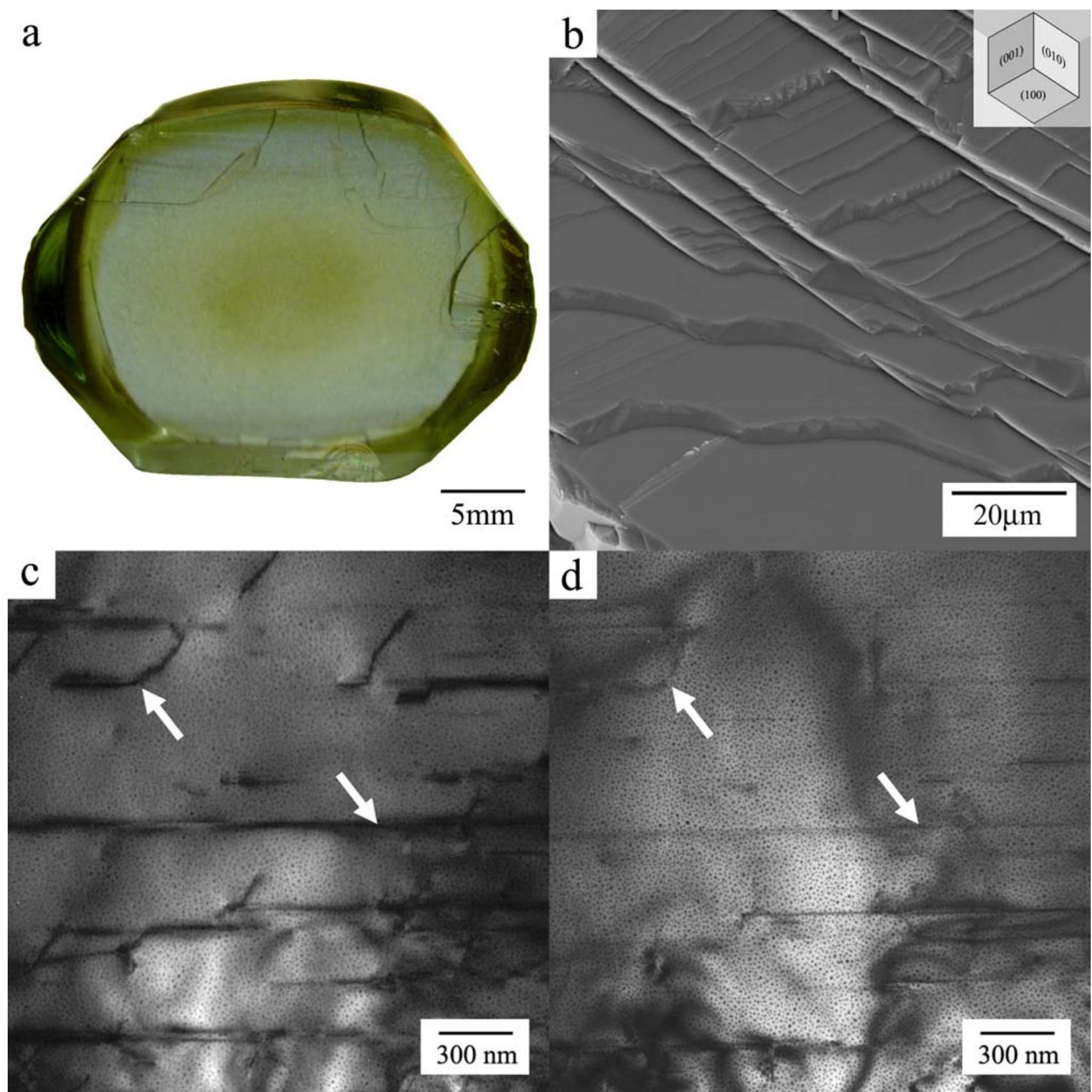

Figure 1 (a) Image of $\mathrm{YVO}_{4}$ crystal showing a central region of discoloration. (b) SEM image of a cleavage surface of $\mathrm{YVO}_{4}$. (c) Bright-field image showing dislocation structures in $\mathrm{YVO}_{4}$ (d) Brightfield image oriented so that certain dislocations are "invisible" (arrows indicate equivalent regions). 